\title{
Innovation et apprentissage organisational : quatre modèles de dialogue entre la logique dominante et la logique nouvelle
}

René Bouwen et Ronald Fry

\section{OpenEdition}

Journals

Édition électronique

URL : http://journals.openedition.org/communicationorganisation/1623

DOI : 10.4000/communicationorganisation. 1623

ISSN : 1775-3546

Éditeur

Presses universitaires de Bordeaux

Édition imprimée

Date de publication : 1 mai 1993

ISSN : 1168-5549

Référence électronique

René Bouwen et Ronald Fry, «Innovation et apprentissage organisational : quatre modèles de

dialogue entre la logique dominante et la logique nouvelle », Communication et organisation [En ligne],

3 | 1993, mis en ligne le 19 décembre 2012, consulté le 05 mai 2019. URL : http://

journals.openedition.org/communicationorganisation/1623; DOI : 10.4000/

communicationorganisation. 1623

Ce document a été généré automatiquement le 5 mai 2019.

(C) Presses universitaires de Bordeaux 


\title{
Innovation et apprentissage organisational : quatre modèles de dialogue entre la logique dominante et la logique nouvelle
}

\author{
René Bouwen et Ronald Fry
}

\section{NOTE DE L'ÉDITEUR}

Cet article est d'abord paru dans International Studies of Managment and Organization, vol. 21, n 4, 1991. Il est traduit et publié avec la permission de l'éditeur, M.E. Sharpe Inc. Armonk, New York, 10504

\section{Introduction : l'importance de l'innovation organisationnelle}

1 Dans toute l'histoire de l'humanité, jamais autant d'organisations sociales ou commerciales n'ont eu à composer avec autant de changements fondamentaux, à un rythme aussi croissant de changement. Les pressions des environnements économique et politique, tout comme les pressions internes provoquées par l'apparition de technologies nouvelles et l'évolution des attitudes de leurs membres, menacent la survie même de nombreuses organisations. Comprendre et orienter le processus de renouveau ou d'innovation organisationnelle est devenu une aptitude vitale que les organisations doivent acquérir. L'innovation ne se limite d'ailleurs pas à l'arrivée de nouvelles technologies des produits. Les retournements, fusions, restructurations, programmes de qualité totale et d'amélioration continue, projets de globalisation et autres, sont à l'avantscène de nombreuses transformations actuelles. Ils exigent de l'organisation la capacité d'apprendre comment traduire les idées et les intentions en actions innovatrices. 
2 Nous définirons ici l'innovation, à la suite de Van de Ven (1986), comme «le développement et l'implantation de nouvelles idées par des gens qui, dans le temps, s'engagent dans des transactions avec d'autres personnes au sein d'un ordre institutionnel ». L'accent est donc mis sur les processus d'interactions qui surviennent parmi des individus à propos de nouvelles idées dans un contexte organisationnel. C'est ce que nous appelons le processus d'innovation organisationnelle. Notre intérêt porte plus particulièrement sur les différentes manières par lesquelles les gens s'organisent autour de nouvelles tâches et sur les conséquences de ces efforts sur les processus organisationnels après l'implantation de l'innovation. En d'autres termes, qu'est-ce que l'organisation a appris de son effort d'innovation et comment l'a-t-elle appris. L'apprentissage organisationnel sera pour sa part défini comme l'augmentation de la capacité processuelle d'innover, dans l'avenir, au sein de cette même organisation (Argyris et Schon, 1978). Notre principal postulat est ici que la qualité du dialogue qui a lieu pendant l'effort d'innovation définira la qualité de l'apprentissage organisationnel.

3 Nous présenterons d'abord un cadre d'analyse qui conçoit ce dialogue comme une manière d'explorer, de faire la médiation et de composer avec ce que nous croyons être le cœur d'une situation d'innovation: les tensions entre les logiques organisationnelles dominante et nouvelle. Nous exposerons ensuite, à partir d'analyses de terrains, quatre stratégies distinctes qui composent avec ces tensions. Les implications pour l'apprentissage organisationnel seront finalement discutées.

\section{L'innovation organisationnelle comme processus social}

4 La tentative de comprendre l'innovation organisationnelle comme un processus d'interaction sociale reçoit beaucoup d'appuis dans la littérature sur l'innovation. Il ressort de cette dernière que les facteurs critiques stimulant ou entravant l'innovation dans les grandes organisations relèvent d'un manque de processus de communication internes et externes adéquats, d'une carence d'équipes de travail interdisciplinaires, de la bureaucratisation, d'une culture de "procédures standardisées", de systèmes de rétribution inappropriés et des attitudes de gestionnaires (Rothwell, 1977). La qualité des processus de communication internes est soulevée à maintes reprises comme un élément décisif des innovations réussies (Maidique, 1980; Tushman, 1979). Robert (1977), par exemple, s'est attardé à l'importance des rôles organisationnels dans les processus de communication. Burgelman (1984) a, quant à lui, mis l'accent sur la capacité des gestionnaires à conceptualiser les activités engagées dans les projets entrepreneriaux internes. La fragmentation organisationnelle (Kanter, 1983), contrairement aux formes organiques ou intégrées d'organisation, est largement contre-productive pour l'innovation.

5 L'attention accordée à l'importance de la communication et de l'interaction dans le processus d'innovation a donné naissance à une nouvelle perspective sur les processus organisants eux-mêmes. Selon cette dernière, il est préférable de concevoir l'organisation comme un processus continu de construction sociale basée sur les significations que les gens tirent des interactions et des dialogues sur le travail. Dans ce paradigme constructioniste (Gergen, 1978), l'organisation n'est plus considérée comme une entité donnée et extérieure à soi qui reste à découvrir par l'emploi d'instruments objectifs et 
scientifiques appropriés. Une organisation apparaît plutôt comme une réalité sociale négociée qui comporte un certain niveau de partage de significations. À travers la communication et la négociation, un processus continu de construction sociale a cours parmi les acteurs dans le but d'atteindre le degré de confiance qui rend possible une action commune (Gray et al., 1985). Par conséquent, le discours social est un processus clé à comprendre. La qualité du dialogue devient un facteur critique dans la construction des processus organisant (Weick, 1970). Ce paradigme semble particulièrement approprié à la description des expériences auxquelles les projets d'innovation donnent lieu.

\section{L'expérience de l'innovation : la tension entre la logique dominante et la logique nouvelle}

6 Pour les acteurs engagés dans des projets d'innovation, le changement est souvent vécu comme une perturbation des pratiques organisationnelles en cours. "Nous devons repartir à zéro ", "C'est vraiment la guerre ", "Personne n'a de temps pour personne ", "C'est très excitant mais aussi très exigeant ", sont autant d'expressions fréquemment entendues dans ces circonstances. Tandis que le contenu de l'innovation - le nouveau produit ou service, ou la nouvelle configuration - traverse plusieurs étapes de développement, les pressions sociales parmi les groupes concernés prennent des formes différentes. Trois aspects du tissu social quotidien de la vie organisationnelle sont au centre du processus d'innovation: la façon dont la raison d'être et la valeur des activités sont établies et maintenues; la manière dont la nouveauté est introduite et transformée en idées attrayantes et propres à générer une action; la manière dont la transition de l'ancien au nouveau est effectivement réalisée (Bouwen et Fry, 1988). L'équilibrage ou l'alignement de ces trois thèmes fondamentaux crée une tension constante entre la logique dominante (celle des manières actuelles de faire, des traditions, des valeurs et coutumes historiques) et la logique nouvelle (celle des opportunités, des tentatives, des expérimentations, etc).

7 En définissant et en agissant sur des aspects spécifiques de son environnement interne et externe, l'organisation développe une logique dominante (Prahalad et Bettis, 1986). Cette logique organisationnelle est composée des pratiques de gestion, des compétences particulières qu'exercent des acteurs clés, des expériences passées conservées dans l'organisation et dans les modèles cognitifs employés pour définir les problèmes d'une manière spécifique. La logique dominante d'un département voué à la recherche, par exemple, peut être décrite comme étant essentiellement de nature "académique »: la curiosité scientifique et la découverte de questions stimulantes peuvent entraîner la coordination du travail d'une équipe de recherche. Un sentiment de continuité émerge de la reproduction des modèles scientifiques d'enquêtes. Lorsqu'une telle logique dominante est confrontée à la tâche de réduire les cycles d'introduction de nouveaux produits ou d'augmenter la proportion des designs conviviaux, de nouvelles questions sont soulevées qui remettent en question les pratiques « actuellement acceptables » dans chacun de ces domaines de continuité ci-haut mentionnés. Lorsque l'on introduit de nouvelles manières de traiter les idées ou de gérer le changement, c'est la continuité qui est remise en cause. Là où la continuité est une préoccupation, nous devons réexaminer le rythme de la transformation ou l'équilibre entre les résultats à court terme et les visions d'avenir. Une situation d'innovation est ainsi caractérisée par une multiplicité de cadres de référence ou de perspectives émergeant pour questionner la logique dominante qui assure les pratiques 
et expériences existantes. La manière dont ces perspectives anciennes et nouvelles sont soulevées, débattues, comparées, analysées, comprises et développées constitue le point de mire crucial de cet essai. La figure 1 illustre un schéma des actions survenant lors de la «situation d'innovation ».

Figure 1 : Séquence des actions survenant dans la construction d'un projet d'innovation

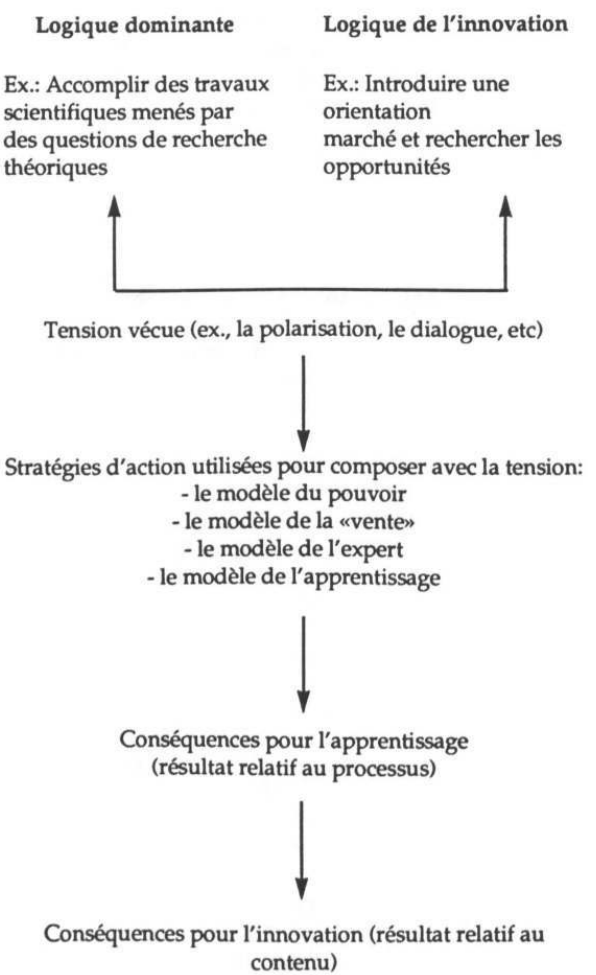

8 La tension créée entre la logique dominante de l'organisation et la logique nouvelle peut être vécue de façons très différentes (Bouwen, 1990). De même, la recherche sur le terrain permet de dégager diverses stratégies d'action destinées à gérer ou composer avec ces tensions. La suite de cet article s'attardera à décrire quatre principales stratégies d'action vouées à provoquer les innovations organisationnelles. Les caractéristiques, les conséquences et les conditions propices à l'émergence de ces différentes configurations conduisent à ce que nous appelons des trajectoires d'innovation. Celles-ci impliquent des conséquences globales distinctes quant à l'apprentissage organisationnel et au potentiel futur d'innovation.

\section{L'étude}

Les stratégies d'action discutées ici sont tirées d'une série d'études de cas. Sept projets d'innovations menés au sein de grandes organisations et six autres au sein d'entreprises plus petites ou naissantes ont été étudiés. Des entrevues ont été réalisées avec huit à douze personnes, à trois reprises chacune, sur des périodes allant de huit à dix-huit mois. Des observations participantes, des procès-verbaux de réunions et autres documents pertinents ont été employés à titre de sources additionnelles de données. Nous avons analysé les transcriptions d'entrevues (plus de 600 pages) afin de développer les concepts propres à rendre compte des similitudes et des différences parmi les treize sites à l'étude. 
Des analyses thématiques ont permis d'identifier des "patterns » communs au sein de l'ensemble des cas. Nous avons décrit ailleurs un modèle de "projets d'innovation » suggérant que les innovations organisationnelles traversent cinq phases consécutives (Bouwen et De Vish, 1989). Nous nous attarderons donc plutôt ici à l'ensemble des stratégies, relevées dans les cas étudiés, qui composent avec la tension entre la logique « dominante » et la logique « nouvelle ». Nous examinerons en particulier le succès de ces stratégies à cet égard.

\section{Les stratégies d'action dans l'innovation organisationnelle}

10 Les études de cas ont permis de relever quatre types distincts de «trajectoires d'innovation ", ou de stratégies d'action, pour communiquer et générer un dialogue sur les logiques anciennes et nouvelles. Bien qu'aucune des situations n'ait pu être entièrement caractérisée par une stratégie unique, nous avons choisi de les présenter ici (tableau 1) sous forme de types idéaux afin d'en clarifier la présentation et la discussion.

11 Ces quatre «trajectoires » peuvent être utilement comparées entre elles sur la base de huit facteurs (voir la colonne de gauche du tableau 1).

12 D’abord, le rôle du « leadership » peut y être assez différent. Nous songeons ici à celui ou à ceux qui jouent un rôle déterminant en rattachant le projet au reste de l'organisation (Burgelman, 1984). La nature générale des processus de communication entre les parties concernées est ici étroitement associée au facteur de "leadership ». Le degré d'unidirectionnalité ou de bidirectionnalité des interactions est une caractéristique clairement distinctive du dialogue. Les critères servant à la prise de décision tout au long du processus sont aussi très différents et reflètent les relations de pouvoir que l'on retrouve entre les parties prenantes et ceux qui ont des intérêts dans l'organisation.

13 La tension principale, ou le facteur critique de succès d'une stratégie, est une autre caractéristique qui varie considérablement d'un cas à l'autre. On peut également observer différents critères d'efficacité et de progrès dans les cas analysés. La succession des actions posés peut aussi prendre des formes diverses. Les formes que prennent les séquences de conduites donnent un aperçu de l'ensemble de ce processus. Un aspect frappant des différences est l'effet de l'apprentissage ou l'adaptation qui survient dans l'organisation. La question soulevée ici concerne la base même ou le mécanisme entraînant le changement dans l'organisation. On peut y voir à l'œuvre différents principes d'apprentissage. Finalement, le succès de chacune des stratégies d'actions employées suppose des conditions propices qui lui sont également spécifiques. Ces circonstances peuvent être caractérisées sur un continuum allant des situations où s'exercent de fortes contraintes à celles où règne un haut degré d'interdépendance et de créativité.

14 À partir de ces huit caractéristiques, nous formulons quatre "modèles " de stratégies d'action. Toutes ces stratégies peuvent produire un effet d'innovation à court terme. Elles se différencieront principalement par leurs conséquences sur le traitement de l'innovation dans l'avenir. C'est ce que nous appelons « l'apprentissage organisationnel ».

15 Nous décrirons ici chacun des quatre modèles - le modèle du pouvoir, de la "vente ", de l'expert et de l'apprentissage-confrontation- à l'aide d'une illustration typique tirée des cas que nous avons étudiés. Le tableau 1 donne une vue d'ensemble des différents aspects soulevés dans chacun des quatre modèles. 


\section{Comparaison des caractéristiques de quatre modèles d'innovation (tableau 1)}

Tableau 1

\begin{tabular}{|c|c|c|c|c|}
\hline & $\begin{array}{l}\text { Le modèle du } \\
\text { pouvoir }\end{array}$ & $\begin{array}{l}\text { Le modèle de } \\
\text { la vente }\end{array}$ & $\begin{array}{l}\text { Le modèle de } \\
\text { l'expert }\end{array}$ & $\begin{array}{l}\text { Le modèle de } \\
\text { l'apprentissage } \\
\text { confrontation }\end{array}$ \\
\hline $\begin{array}{l}\text { 1. Le rôle du } \\
\text { leader }\end{array}$ & $\begin{array}{l}\text { La figure d'auto- } \\
\text { rité qui a accès } \\
\text { aux ressour-ces }\end{array}$ & $\begin{array}{l}\text { Le «vendeur» } \\
\text { employant une } \\
\text { approche en } \\
\text { douceur }\end{array}$ & $\begin{array}{l}\text { Le détenteur du } \\
\text { savoir et du } \\
\text { pouvoir d'expert }\end{array}$ & $\begin{array}{l}\text { Le efacilita-teur } \\
\text { doté d'une con- } \\
\text { naissance du } \\
\text { processus }\end{array}$ \\
\hline $\begin{array}{l}\text { 2. Caractéris- } \\
\text { tiques du } \\
\text { processus }\end{array}$ & $\begin{array}{l}\text { L'imposition, le } \\
\text { changement par } \\
\text { décret }\end{array}$ & $\begin{array}{l}\text { La persuasion } \\
\text { utilisant tous les } \\
\text { moyens d'in- } \\
\text { fluence }\end{array}$ & $\begin{array}{c}\text { Les étapes de la } \\
\text { résolution de } \\
\text { problème ou la } \\
\text { rationalité dans } \\
\text { le traitement de } \\
\text { l'information }\end{array}$ & $\begin{array}{l}\text { La facilitation de } \\
\text { la confrontation; } \\
\text { la restructu- } \\
\text { ration cognitive } \\
\text { et émotionnelle } \\
\text { des deux parties }\end{array}$ \\
\hline $\begin{array}{l}\text { 3. Critères de } \\
\text { prise de décision }\end{array}$ & $\begin{array}{l}\text { Définis par les } \\
\text { intérêts et le } \\
\text { pouvoir }\end{array}$ & $\begin{array}{l}\text { Conserver l'at- } \\
\text { tention; la visibi- } \\
\text { lité continue }\end{array}$ & La rationalité & $\begin{array}{l}\text { La consultation } \\
\text { et la validation } \\
\text { consensuelle }\end{array}$ \\
\hline $\begin{array}{l}\text { 4. Tension } \\
\text { principale dans } \\
\text { le processus }\end{array}$ & $\begin{array}{l}\text { Contrôle des ré- } \\
\text { sultats et des } \\
\text { ressources }\end{array}$ & $\begin{array}{l}\text { Caractère inno- } \\
\text { vateur de l'alter- } \\
\text { native proposée }\end{array}$ & $\begin{array}{l}\text { Discrétion dans } \\
\text { la participation }\end{array}$ & $\begin{array}{l}\text { Précocapationsde } \\
\text { toutes les parties } \\
\text { concernées }\end{array}$ \\
\hline $\begin{array}{l}\text { 5. Critères } \\
\text { d'efficacité }\end{array}$ & $\begin{array}{l}\text { Résultats ra- } \\
\text { pides, financiers } \\
\text { et/ou tech- } \\
\text { niques }\end{array}$ & $\begin{array}{l}\text { Taux d'adoption } \\
\text { et d'adhésion à } \\
\text { la nouveauté }\end{array}$ & Résultat rationnel & $\begin{array}{l}\text { «Sens commun } \\
\text { interprétations } \\
\text { communes }\end{array}$ \\
\hline $\begin{array}{l}\text { 6. Séquence des } \\
\text { patterns d'action }\end{array}$ & $\begin{array}{l}\text { Commence- } \\
\text { ment et recom- } \\
\text { mence-ment; ré- } \\
\text { gression }\end{array}$ & $\begin{array}{l}\text { Planification co- } \\
\text { hérente (avan- } \\
\text { cées et d'arrêts) }\end{array}$ & $\begin{array}{l}\text { Processus linéai- } \\
\text { re respectant } \\
\text { une suite d'éta- } \\
\text { pes }\end{array}$ & $\begin{array}{l}\text { Expérimentation } \\
\text { / évaluation et } \\
\text { questionnement } \\
\text { : cycle d'appren- } \\
\text { tissage }\end{array}$ \\
\hline $\begin{array}{l}\text { 7. Effet sur } \\
\text { l'apprentissage } \\
\text { organisationnel }\end{array}$ & $\begin{array}{l}\text { Conformité et } \\
\text { adhésion passive }\end{array}$ & $\begin{array}{l}\text { Imitation et a- } \\
\text { doption }\end{array}$ & $\begin{array}{l}\text { Apprentissa-ge } \\
\text { cognitif par la } \\
\text { compréhension }\end{array}$ & $\begin{array}{l}\text { Communication } \\
\text { et orien-tation } \\
\text { sur la base de } \\
\text { données valides }\end{array}$ \\
\hline 8. Conditions & $\begin{array}{l}\text { Situation de cri- } \\
\text { se; manque de } \\
\text { temps; dépen- } \\
\text { dance voulue }\end{array}$ & $\begin{array}{l}\text { Indifférence du } \\
\text { groupe concer- } \\
\text { né; enjeu d'un } \\
\text { seul coté }\end{array}$ & $\begin{array}{l}\text { Situation prévi- } \\
\text { sible et forte- } \\
\text { ment structurée }\end{array}$ & $\begin{array}{l}\text { Interdépendanc } \\
\text { e indispensable } \\
\text { a l'atteinte des } \\
\text { objectifs }\end{array}$ \\
\hline
\end{tabular}

\section{Le modèle du pouvoir/influence}

16 FOOD est une entreprise de restauration rapide, division d'un groupe de distribution alimentaire. L'innovation consista à y introduire une nouvelle formule de restaurant, de type « classique ». L'idée de cet innovation est venue à un cadre supérieur au cours d'un voyage à l'étranger. Un franchiseur fut choisi pour soutenir le projet en fournissant expertise et formation. On engagea et forma un dirigeant local et quelques employés pour «exécuter» les idées développées par la société mère et le franchiseur. On donna l'instruction aux employés de mettre l'accent sur les valeurs centrales de la haute direction de la société mère: produire un retour sur les investissements, respecter des budgets préétablis et viser le succès à court terme. L'expérience a été vécue comme un lourd fardeau pour ceux et celles qui y furent engagés, à commencer par le dirigeant local. Lorsque les problèmes surgirent, on embaucha un nouveau dirigeant. Le roulement de personnel continua toutefois de s'accentuer et avec l'emploi de personnel inexpérimenté les coûts ne cessèrent de s'accroître.

17 Les employés interviewés dans le nouveau restaurant ont rapporté avoir été aux prises avec un fossé s'élargissant sans cesse entre les exigences de l'organisation mère et les contraintes du service à la clientèle, de l'équipe de travail et des responsabilités 
personnelles au sein de l'unité locale. Au bout d'une année, le nouveau restaurant fut revendu au franchiseur.

Dans ce cas, nous pouvons constater que la direction agit à partir d'une position d'autorité. Tout l'effort d'innovation est introduit et dirigé depuis le sommet. Il est compréhensible, dès lors, que la direction se réfère à ses propres critères de réussite, empruntant une orientation menée par la gestion financière (l'ancienne logique). L'innovation est décrétée du sommet sans qu'aucun processus de développement interne ne soit entrepris. Le dirigeant local du projet joue le rôle de l'exécutant des directives provenant de la haute direction. Il vit une forte tension entre les demandes de l'organisation et celles qui émergent du nouveau projet. L'expertise dans son champ d'activité, le travail d'équipe et l'orientation vers le client doivent être nourris afin de motiver le personnel autant que les clients eux-mêmes. Il n'y avait ni temps ni intérêt au sein de la haute direction pour s'attarder à ces aspects. S'il s'était avéré possible d'assurer le bon fonctionnement d'un supermarché en recourant à des procédures standardisées, en faire autant avec ce nouveau type de restaurant devenait une toute autre affaire. Lorsque les problèmes surgissent, un nouveau départ est repris avec le remplacement du gestionnaire et de certains employés. Le nouveau dirigeant fait toutefois face aux mêmes problèmes. La décision de vendre le nouveau restaurant était donc peut-être «la seule possible » dans ce contexte. À titre de résultat cependant, l'innovation a entrainé fort peu de changement dans cette organisation. Le style de direction ne valorisant que l'adhésion passive aux volontés de l'organisation mère, il était très difficile pour cette grande organisation d'intégrer les exigences du nouveau projet.

Il est clair que dans ce cas les effets d'apprentissage sont plutôt limités. Cette organisation a peut-être simplement appris comment ne pas innover. Il est probable que les personnes concernées aient interprété ces événements comme reconfirmant la valeur des anciennes pratiques ou qu'elles en aient tiré des leçons du type «une promotion s'obtient par le respect des prévisions budgétaires » ou encore « le blâme pour les problèmes survenus sera porté par les individus qui seront renvoyés ».

La stratégie correspondant au modèle du pouvoir pourrait éventuellement s'avérer appropriée dans les situations où il n'y a pas d'attentes explicites en ce qui a trait à l'apprentissage et où l'implantation n'implique pas de transformations significatives des pratiques en cours. On doit probablement s'attendre, par ailleurs, à ce que ce type de stratégie émerge dans les situations de crise où lorsque le temps fait sérieusement défaut. À partir des autres cas que nous avons étudiés, nous constatons que ce modèle est employé assez couramment; il découle d'une logique dominante autoritariste. La plupart du temps, toutefois, la logique de l'innovation est très différente. On comprend ainsi comment il peut être difficile pour une organisation autoritaire d'innover.

\section{Le modèle de la « vente »}

21 BANK est une caisse d'économie de taille moyenne introduisant une nouveau système de classification pour ses clients. Le directeur des services informatiques, qui a pris cette initiative, est, en outre, le chef d'un groupe de travail sur ce projet d'innovation formé de représentants des divisions locales et les départements centralisés. La participation des agences locales est cruciale puisque ces dernières doivent fournir les données et en constituent les principaux utilisateurs dans leurs contacts avec les clients. Le département des services informatiques désire avoir un contrôle sur la constitution du 
système afin que celui-ci ne puisse être manipulé par les agents locaux. Le dirigeant du projet est considéré comme le champion de ce nouveau système; avec l'aide de son personnel technique, il a lui-même développé les spécifications du système. Il consacre beaucoup d'énergie à faire émerger ce qu'il appelle une orientation vers un consensus au sein du groupe de travail sur le projet. Il mène activement les discussions, mettant l'accent sur les principes, soulignant les intérêts communs des membres, insistant sur le fait que le nouveau système doive être à la fois commercialement efficace (répondant aux besoins des clients) et sûr (tenant compte des règles de sécurité destinées à prévenir les mauvais usages). Il reconnait la contribution et les efforts de tous les membres, crée un climat positif dans les rencontres et maintien continuellement un équilibre entre susciter la participation des membres et les objections et sauvegarder les caractéristiques du système qu'il juge essentielles. Il demeure le membre de l'équipe le mieux informé, fournit toutes les informations à caractère technique, fait les calculs nécessaires à l'évaluation des alternatives et structure constamment les réunions, tout en mettant l'accent sur l'importance du groupe de travail. L'un des membres du groupe a décrit ainsi sa conduite : «Il est compétent, très intelligent et discute aisément. Cela lui confère une auréole d'excellence. Mais il est très difficile de l'arrêter ».

Par ailleurs, le dirigeant du projet minimise le caractère innovateur du système en affirmant qu'il ne constitue qu'une formalisation de pratiques qui, dans les faits, avaient cours depuis des années déjà. Il est très soucieux de ne pas avancer trop rapidement. Un membre du groupe appartenant à une agence externe parle positivement du groupe de travail, considérant qu'il facilite le fonctionnement des services centralisés dans ses rapports avec les agences locales. Comme premier résultat, le groupe de travail fut institutionnalisé à titre de département " d'organisation », voisin du président lui-même et le dirigeant du projet fut nommé conseiller immédiat de ce dernier. Le second résultat de ce processus s'est traduit par la réception favorable qu'a reçu le nouveau système dans l'ensemble de l'organisation et le développement d'autres instruments de coordination voués à « soutenir » et à « guider » les divisions locales.

Dans cette trajectoire d'innovation, le rôle du dirigeant de projet est tout à fait central. Agissant comme une sorte de "vendeur à l'approche douce ", il insiste sur le fait que le consentement des usagers doit être au cœur des préoccupations et il utilise une attention et une persuasion soutenues pour obtenir les actions nécessaires. Tout le travail du groupe de projet peut être vu comme une action pour aider l'organisation à digérer progressivement l'introduction de mécanismes de contrôle et de centralisation de l'information. Beaucoup d'attention est consacrée à convaincre les agents locaux que le projet va dans le sens de leurs intérêts. Le critère employé pour mesurer le progrès accompli et pour aller de l'avant est l'intérêt et la coopération obtenus des parties concernées. Toutes les objections et réactions sont prises en considération mais les principes originaux demeurent inchangés. La promotion éventuelle du dirigeant de projet facilite le consentement mais illustre, ce faisant, comment le mécanisme agissant dans cette stratégie constitue davantage un consentement bienveillant qu'une contribution critique et un apprentissage personnel.

Bien que le résultat soit plutôt une réussite du point de vue de l'organisation, l'effort d'apprentissage de l'organisation elle-même est plutôt réduit dans ce cas. Le processus a créé plus l'adhésion et le consentement passif que le partage de la responsabilité et la participation active. L'apprentissage organisationnel, entendu comme une capacité plus grande d'innover et de changer, ne sera pas très appréciable pour cette organisation au 
terme du projet. Il est possible que l'essentiel du sens créé rétrospectivement par les participants concerne le dirigeant du projet, c'est-à-dire qu'il porte sur la manière de devenir le champion d'une vision et de se positionner pour un poste de haut niveau.

\section{Le modèle de l'expert}

25 Le projet d'innovation réalisé chez COMMUNICATIONS porte sur l'introduction d'un système de conception et de production assistées par ordinateur dans le département de design et développement d'une entreprise de taille moyenne spécialisée dans la fabrication d'équipement électronique. Ses produits sont hautement innovateurs par rapport aux normes de son marché. L'organisation a un haut niveau de spécialisation des fonctions et un respect de la hiérarchie, mais conserve les caractéristiques d'une entreprise familiale ainsi qu'une préoccupation aiguë pour la qualité. La nécessité de raccourcir les cycles de conception et de production a contraint l'organisation à reconsidérer l'introduction d'une technologie de l'information dans ce département ; une tentative menée dans ce sens cinq ans plus tôt s'était soldée par un échec. Cette fois, un responsable de projet fut embauché spécialement pour travailler à l'implantation.

Le responsable du projet suivit, tout au long du processus, deux principes; ce devait être une approche intégrée: toutes les fonctions touchées par le projet doivent y être associées, de même que tous les niveaux hiérarchiques (les concepteurs tout autant que le directeur). Il décrivit lui-même son approche :

«Nous formulons toujours une première proposition. Celle-ci est expliquée aux concepteurs : par exemple, ma proposition initiale concernant la table à dessin fut d'opter pour un modèle électrostatique de 200 points. Nous leur avons présenté des exemples ainsi que les différentes options et leur avons demandé : «Regardez, voici les différentes alternatives et voici les possibilités de ce système. Qu'est-ce qui est le plus approprié à votre situation particulière? » Si vous demandez simplement aux gens qu'ils vous proposent des alternatives, vous n'obtiendrez aucune réponse. Ils n'y comprennent rien, vous devez les leur expliquer. Après les explications, nous avons considéré toutes les options. Ils ont pu intervenir et ont abandonné très rapidement la proposition de la table de 200 points, mais seulement après que je leur eus donné l'information appropriée. »

Le responsable du projet collabora continuellement avec le directeur du laboratoire de conception et le gestionnaire des services informatiques. Le processus de communication entre les différentes parties concernées était plutôt linéaire et axé sur des problèmes spécifiques. Toutes les questions, comme la définition et la clarification des problèmes ou la proposition et l'évaluation des alternatives, étaient traitées étape par étape au fil des réunions du groupe associé au projet. On a également maintenu, en tout temps, des contacts fréquents et des échanges d'informations avec les usagers. Les principaux participants concernés développèrent un engagement grandissant dans le traitement des difficultés techniques et le choix des critères d'évaluation. Chez les concepteurs, on a même observé un empressement à commencer à travailler avec le nouveau système. Le responsable du projet rédigea un rapport de 250 pages sur l'ensemble du processus d'examen de la problématique. Les trois alternatives retenues furent longuement décrites à partir des informations recueillies lors des nombreuses réunions et des contacts personnels. On laissa au directeur général la décision définitive quant au système à choisir ; il s'agissait pour lui de négocier avec les représentants des trois fournisseurs en lice. Cette dernière étape fut qualifiée de "politique ». Le groupe associé au projet avait alors remis le choix final au directeur afin qu'il puisse « jouer le jeu de la négociation ». 
28 Le modèle de stratégie d'action mis en œuvre dans ce cas respecte les étapes d'un processus rationnel de résolution de problème et de prise de décision, avec une participation étroite de tout le personnel nécessaire. Le responsable de projet est embauché en sa qualité d'expert. Il recueille toute l'information utile à la réalisation d'une analyse fonctionnelle du problème qui soit complète et acceptée par les personnes concernées. Il joue un rôle d'instructeur, expliquant à maintes reprises toutes les implications possibles des questions soulevées. Les décisions sont prises après des discussions essentiellement axées sur les tâches et tenant compte de tous les aspects de l'analyse fonctionnelle. La participation des usagers à l'ensemble de ce processus aurait possiblement pu être réduite. Cependant, à cause du rejet précédent d'une innovation similaire, le responsable du projet a été très attentif à faire participer autant que possible les usagers au processus. Si d'un point de vue purement rationnel cette participation n'était pas en mesure de contribuer d'une manière significative a la conception du nouveau système, elle pouvait en revanche faciliter considérablement son implantation, dans la mesure où les usagers sentaient leurs besoins pris en compte. Le critère de réussite, dans cette stratégie d'action, est le fait que chacun puisse suivre et comprendre l'objet des décisions et soit convaincu que la décision prise sera la plus rationnelle. En conséquence, les gens se sont engagés dans un apprentissage commun des problèmes techniques avec lesquels ils doivent composer. Il s'agit là d'un apprentissage de niveau cognitif. Toutefois, rien n'indique qu'un apprentissage au niveau social ou organisationnel ait eu lieu. Le responsable du projet conduisit le processus sur la base de son expertise. On peut considérer ce cas comme un exemple de gestion de projet réussie.

\section{Le modèle de l'apprentissage/confrontation}

29 Filiale belge d'une organisation multinationale américaine œuvrant dans le domaine des technologies de pointe, la compagnie MATERIALS produit des matériaux isolants pour câbles de télécommunication et autres canalisations. L'entreprise a connu une croissance importante au cours des vingt dernières années (environ $20 \%$ ), mais a vu son développement perturbé dans les années 80 par la crise économique généralisée, l'expiration d'un brevet d'invention et la fragmentation de son marché. Ces événements l'ont contrainte à devenir plus flexibles et orientée davantage vers ses clients. Au cours de la période de croissance, une culture de l'informel, de l'individualisme, de la responsabilité et de la créativité s'y était développée. L'organisation devait maintenant faire face aux contraintes extérieures et aux changements technologiques.

Ce projet d'innovation pris d'abord la forme de deux efforts distincts. D'une part, il était devenu nécessaire de procéder à l'installation de systèmes automatisés de manipulation des matériaux ainsi qu'à l'implantation d'un programme de contrôle de la qualité dans l'ensemble de l'entreprise. D'autre part, la direction au niveau local lança ce qu'elle appelait un projet de développement organisationnel destiné à repenser l'organisation interne de la manufacture. Le projet éventuel, développé sur une période d'environ quatre ans et comportant plusieurs épisodes, est trop complexe pour être décrit adéquatement dans les limites de cet article. Nous devrons donc nous contenter ici de décrire la position des personnes clés, leur conduite et le résultat général du processus.

31 À la suite d'une assemblée tenue à l'extérieur du site de l'entreprise, une série de réunions hebdomadaires débuta pour discuter des deux projets de changements de façon coordonnée. Un énoncé général de la mission fut formulé ainsi : «Notre objectif est 
d'obtenir une organisation (des personnes, des politiques et des instruments) efficace en terme de service à la clientèle (délai de livraison et qualité), de contrôle des coûts (du fonds de roulement et des frais généraux) et d'intégration des produits et des processus nouveaux » Graduellement, au fil de séances, émergea l'idée de doter l'organisation de la manufacture, d'un tableau des fonctions et des tâches, dans lequel toutes les activités (le "quoi ») pourraient être assignées à une personne ou un groupe spécifique (le "qui »). Remplir le tableau impliqua un processus crucial de prise de décision. On avait en effet jugé que la tâche d'assigner des noms aux nouvelles positions revenait au groupe et, manifestement, ce processus produisit beaucoup de tensions et de confrontations. Le directeur de production en décrivit ainsi un moment décisif :

Finalement monsieur $\mathrm{X}$ se leva et se proposa lui-même ouvertement comme candidat. Je ne pouvait pas être d'accord avec cela et je l'ai dit. Je lui proposai de conduire plutôt une autre ligne de production. Les autres participants hésitèrent à faire des propositions supplémentaires. Je formulai alors publiquement ma proposition et inscrivis quelques noms sur l'organigramme. Dès cet instant, il y eu un soulagement général au sein du groupe. J'ai alors quitté la salle pour un moment. Lorsque j'entrai à nouveau, $\mathrm{Y}$ fut le premier à répondre en disant : « Je suis cette proposition. » D'autres noms furent ensuite ajoutés au cours de cette réunion. En fait, nous devions réfléchir encore beaucoup à ce qui devait être fait. J'ai dû parler à un grand nombre de personnes avant qu'une proposition complète puisse émerger de tout cela. »

$\mathrm{Au}$ terme du processus, cinq nouveaux groupes de produits furent établis sur la base d'une orientation centrée sur l'usine, intégrant du personnel conseil et de service. L'étape suivante importante fut l'implantation du projet au niveau de l'usine et, dans une phase ultérieure, dans les relations avec d'autres développement techniques aussi bien qu'avec le marketing et la recherche. Le projet a réussi à mettre en place des systèmes d'information intégrés pour la circulation des matériaux, étroitement reliés à la réaffectation de personnel aux nouveaux groupes de travail, aux nouveaux principes et aux nouvelles procédures.

Parmi les caractéristiques de base de cette innovation, retenons les suivantes: sa complexité, recouvrant toutes les facettes de l'organisation et poursuivant un objectif en mouvement et en développement constant; un haut degré d'ambivalence chez toutes les personnes engagées dans le processus; un niveau élevé de confrontation; et un degré tout aussi élevé d'engagement et d'ouverture à la discussion sur tous les aspects du projet. La confrontation était manifeste pendant les nombreux contacts et les réunions au cours desquels toutes les dimensions de la restructuration étaient ouvertement discutées. On pouvait aussi observer que les membres groupe discutant du projet prenaient fréquemment ce qu'ils appelaient un «temps d'arrêt», c'est-à-dire une interruption permettant de jeter un regard sur ce qu'ils avaient fait et sur la manière dont ils l'avaient fait. Cela provoqua un second niveau de confrontation et de compréhension portant cette fois sur le déroulement de l'action. Au cours des séances du groupe de discussion sur le projet, la direction jouait essentiellement un rôle de facilitateur. Une personne chargée de la gestion des ressources humaines était membre permanent du groupe de discussion. Elle se donnait pour tâche d'attirer l'attention du groupe sur la nécessité de développer un processus approprié, ou du moins, de réfléchir sur la façon dont le groupe progressait. La confrontation portait donc à la fois sur le contenu des discussions et, à un niveau "méta ", sur le processus de discussion lui-même. Les entrevues réalisées au terme du projet permettent de conclure que les participants effectuaient un apprentissage continu à propos de ce qu'ils faisaient et de la manière dont ils le faisaient. Il est clair, par ailleurs, 
qu'un tel niveau de confrontation exige beaucoup des personnes concernées. Pour certains, les rétroactions continues et la demande incessante de réactions ont été vécues comme beaucoup trop exigeantes. Un ingénieur en procédés, à titre d'exemple, décida de se mettre à la recherche d'un endroit plus paisible, comme il l'admit ouvertement.

La qualité la plus importante de cette trajectoire d'innovation est le caractère réciproque des processus de communication qu'elle met en œuvre. Nous retrouvons les caractéristiques du modèle II d'apprentissage organisationnel proposé par Argyris et Schon (1978) : des propositions concrètes, bien illustrées, émises à titre d'essai et ouvertes à toutes les réactions possibles des participants au dialogue. On assiste à une appropriation collective de la tâche d'interaction et à une influence partagée sur les résultats. La prise de décision se fonde sur la validation consensuelle (Schein, 1969). Il ne s'agit pas de parvenir à un compromis général, mais plutôt à un accord appuyé par tous les parties concernées et cela après que chacune d'entre elles ait eu la pleine opportunité d'exprimer ses préoccupations, obtenu l'assurance d'être comprise et participé à la constitution de la meilleure solution possible.

Le critère de réussite dans un tel effort d'innovation est le degré de partage par toutes les parties concernées d'un "sens commun" ou d'un construit social sur la nouvelle orientation à suivre. Cela signifie que ce processus d'innovation est faiblement déterminé, c'est-à-dire très permissif. Il est constitué essentiellement de questionnements mutuels, d'une recherche des informations et des moyens disponibles, d'expérimentations des alternatives et d'une évaluation continue des conséquences. La qualité la plus importante du résultat de cette démarche réside au niveau du processus: Y a-t-il un véritable engagement des personnes associées au projet et celles-ci fournissent-elles une information valide?

Nous avons appelé cette quatrième trajectoire d'innovation le modèle de l'apprentissageconfrontation pour souligner ses effets d'apprentissage à long terme dans l'organisation, suivant la théorie de l'apprentissage organisationnel d'Argyris, et pour indiquer la part de confrontation que soulèvent les interactions et la communication entre les acteurs qui $y$ sont engagés.

Cette trajectoire est souvent qualifiée par les observateurs extérieurs d'approche "douce ", « orientée vers le client» ou encore "non-directive». Comme on peut le conclure du cas de MATERIALS, il y a en effet beaucoup d'attention portée à la discussion des problèmes avec toutes les parties concernées. On accorde une grande importance à l'engagement personnel et la participation de chacun. On ne s'attend pas à ce qu'un individu se joigne à l'innovation si il ou elle n'a pas fait un choix personnel après avoir eu le temps et l'espace voulus pour exprimer ses idées. Mais cette approche est aussi fondée sur l'accomplissement d'un travail à partir de données valides. Cela signifie que tous doivent mettre en commun l'information dont ils disposent en proscrivant les «jeux » de dissimulation, de charme, d'échange ou d'autres formes de camouflage. Ils doivent s'exprimer ouvertement, ce qui peut impliquer une part de confrontation lorsque l'on discute des erreurs ou des performances inadéquates. Argyris définit l'organisation « apprenante » comme étant celle capable d'identifier et de corriger ses erreurs. Pour y parvenir, il faut que les informations soient disponibles et que les gens se questionnent et se renseignent mutuellement. La confrontation survient donc dans les relations entre les personnes concernées, mais se retrouve également dans la question de l'ouverture et de l'accès aux données nécessaires à une résolution adéquate des problèmes. 


\section{Résumé et implications : les caractéristiques des processus et les indicateurs d'apprentissage}

Cet article avait pour but de nous sensibiliser davantage aux indicateurs de la qualité de la communication ou du dialogue survenant entre des parties engagées dans un projet d'innovation organisationnelle. La littérature actuelle sur l'innovation reconnait un rôle important à la communication. À partir de l'analyse des caractéristiques qu'a revêtues le processus d'innovation dans les cas que nous avons étudiés, nous avons pu dégager des modèles distincts de stratégies d'action (voir le tableau 1 pour une vue d'ensemble). Ces modèles de stratégie produisent des résultats très différents quant à l'apprentissage qu'en retirent les participants sur la manière de mener un effort d'innovation.

La proposition essentielle émanant de cette étude peut être formulée dans les termes suivants : pour retirer des effets d'apprentissage organisationnel durables et à long terme d'un projet d'innovation, une stratégie conséquente avec le modèle de l'apprentissage /confrontation est nécessaire. Les autres modèles d'action peuvent donner quelques résultats à court terme; ils ne peuvent toutefois pas créer un savoir nouveau et partagé. Ils ne peuvent fournir de données valides expliquant pourquoi et comment l'organisation parvient à réaliser au mieux son effort d'innovation.

Dans l'implantation d'une innovation, chacun des quatre modèles peut conduire à une certaine réussite. Nous avons tenté d'identifier plus précisément les conditions dans lesquelles chacun de ces modèles est susceptible d'émerger. Ces conclusions, de même que nos hypothèses de travail, demeurent cependant à évaluer sur une plus large échelle.

Le modèle du pouvoir semble s'appliquer aux situations de crise ou encore à celles où un sérieux manque de temps prive le processus de prise de décision de toutes les étapes appropriées. Dans d'autres situations, certains peuvent choisir consciemment de demeurer dans une situation de dépendance par rapport au système ou à la haute direction. Le modèle du pouvoir peut constituer une stratégie d'action appropriée dans toutes ces situations, pour autant que l'on accepte ses conséquences éventuelles qui sont l'adhésion passive et l'absence de réflexion critique. Des problèmes surgiront dans les efforts de changement organisationnel mûs par l'autorité si l'on attend de ces derniers des effets tels que l'engagement et la responsabilisation.

Le même type de remarque peut être fait à propos du modèle de la « vente » et du modèle de l'expert. De l'approche de la "vente", on doit s'attendre pour l'essentiel à des conduites d'imitations, qui peuvent d'ailleurs suffire à la réussite de l'implantation. L'approche de l'apprentissage, beaucoup plus difficile et exigeante, ne s'impose donc pas ici. Dans une situation bien structurée, où il s'agit surtout de comprendre l'objet du changement, le modèle de l'expert peut être utile. Dans les deux cas, cependant, l'emphase mise sur l'imitation et sur l'aspect cognitif de la résolution de problème limite sérieusement la probabilité que les participants construisent de nouvelles significations sur la manière de se changer et de changer l'organisation à nouveau si cela devenait nécessaire ou s'ils le souhaitaient.

Notre objet a été ici d'identifier et de décrire des modèles d'action propres à caractériser le processus d'innovation. Ces derniers peuvent éclairer l'acteur intervenant dans une situation de changement (le champion, le dirigeant de projet ou le facilitateur) en l'aidant à faire des choix d'une façon plus consciente. La question des conditions favorisant ou entravant l'émergence d'une stratégie d'action spécifique n'a pas trouvé de réponse dans 
ce qui précède. Il est en effet possible que certaines circonstances, comme le type d'innovation, la structure de l'organisation ou le contexte économique influencent l'apparition de stratégies spécifiques. De même, il est possible que le modèle de l'expert soit plus fréquemment employé dans les projets d'automatisation et d'informatisation, que les grandes organisations privilégient le modèle de la persuasion sur celui de l'apprentissage et ainsi de suite. Il est également possible qu'un amalgame de ces modèles surviennent dans le développement d'un projet d'innovation et que certaines phases du projet soient associées à certaines préférences pour un modèle ou un autre. Ces questions devront faire l'objet d'autres travaux. Nous nous sommes limités ici à l'identification de quatre stratégies ou modèles d'action dont nous avons relevé les forces et les faiblesses. Nous croyons que ces modèles devraient se révéler utiles aux innovateurs dans la conception et l'orchestration du changement et de l'apprentissage organisationnels.

Si, par l'emploi des concepts développés dans cette étude, la capacité d'observation des acteurs clés engagés dans une innovation organisationnelle peut être accrue pour leur permettre de discerner les caractéristiques du processus en cours et de les comparer à leurs intentions, alors l'objectif de cet article aura été largement atteint. Un pas crucial pourrait être effectué vers l'accomplissement d'une véritable «organisation apprenante "; une organisation où est constamment présent un discours portant sur ce qu'on apprend de nous-mêmes et de notre capacité collective en travaillant ensemble, une organisation où est encouragée la confrontation entre les pratiques sûres, éprouvées par le temps et les idées, les visions nouvelles.

\section{BIBLIOGRAPHIE}

Argyris, C. \& Schon, D.A., Organizational Learning : A Theory of Action Perspective, Addison-Wesley, Reading, MA, 1978.

Bouwen, R. Organizational Innovation as a Social Construction: Managing Meaning in Multiple Realities. Paper presented at the NJAS Conference, Wassenaar, May 31- June 1, 1990.

Bouwen, R. \& De Visch, J., Innovation Projects in Organisations : Complementing the Dominant Logic by Organisational Learning. Paper presented at the 4th European Congress of Worlk and Organisational Psychology, Cambridge, UK, April 1-12, 1989.

Bouwen, R. \& Fry, R. « An Agenda for Managing Organizational Innovation Development in the 1990s » dans M. Lambrecht (ed.). Corporate Revival, University Press, Leuven, 153-172, 1988.

Burgelman, R., « Managing the Internal Corporate Venturing Process », Sloan Management Review, Winter, 33-18, 1984.

Gergen, K. « Toward Generative Theory », Journal fo Personality and Social Psychology, 36/11, 1344-1360, 1978.

Gray, B., Bougon, M.G. \& Donnellon, A. « Organisations as Constructions and Destructions of Meaning ", Journal of Management, 11, 83-98, 1985.

Kanter, R.M., The Change Masters, G. Allen, London 1983. 
Maidique, M.A., « Entrepreneurs, Champions and Technological Innovation », Sloan Mangement Review, Winter, 59-76,1980.

Prahalad, C.K. \& Bertis, R.A. « The Dominant Logic : A New Linkage between Diversity and Performance », Strategic Mangement Journal, 7, 485-501,1986.

Roberts, E.B. « Generating Effective Corporate Innovation », Technology Review, OctoberNovember, 27-33, 1977.

Rothwell, R. « The Characteristics of Successful Innovations and Technically Progressive Firms », Research and Development Management, 7/3,191-206,1977.

Schein, E.H., Process Consultation : Its Role in Organization Development, Addison-Wesley, Reading, MA 1969.

Tushman, M.L., « Managing Communication Networks in R and D Laboratories », Sloan Management Review, Winter, 37-49, 1979.

Van De Ven, A.H., « Central Problems in the Management of Innovation », Management Science, 32, May, 590-607, 1986.

Weick, K., The Social Psychology of Organizing, Addison-Wesley, Reading, MA, 1970.

\section{RÉSUMÉS}

Ce texte définit l'innovation comme un processus social et le changement comme une tension entre la logique nouvelle et la logique dominante au sein de l'organisation. A partir d'une étude empirique, les auteurs décrivent quatre types de dialogues révélés par quatre stratégies de changement : le changement imposé du sommet (modèle du pouvoir/ influence), le changement par la persuasion (modèle de la vente), le changement qui s'appuie sur la rationalité technique (modèle de l'expert), le changement consensuel émergent (modèle de l'apprentissage/confrontation). Ils en discutent les implications pour l'apprentissage organisationnel.

In this text, innovation is defined as a social process, and change as a tension between the dominant logic and a new logic. Four distinct strategies (or types of dialog) dealing with these tensions are presented from the analyses of field rsearch. The authors describe the power/ influence model, the sales model, the expert model, and the confrontational learning model. They discuss their implications for organizational learning.

\section{AUTEURS}

\section{RENÉ BOUWEN}

René Bouwen est professeur de comportement organisationnel et de dynamique des groupes au Katholieke Universiteit Leuven. Il est docteur en spychologle de la même université. Il fait de la recherche et a publié sur les processus de changement et de l'innovation dans l'organisation, la gestion du conflit et l'efficacité des petits groupes. Il est aussi consultant auprès des organisations industrielles et éducatives.

\section{RONALD FRY}

Ronald Fry est professeur de comportement organisatlonnel au Wheaterhead School of Management. Case Western Reserve University. Cleveland, Ohio (US). Il est docteur de 
psychologie organisationnelle de l'Alfred Sloan School of Management, Massachusetts Institute of Technoloy. Il est consultant et chercheur auprès des organisations dans l'industrie et

l'éducation. Il a publié sur la formation des professionnels, la gestion des ressources humaines, le développement des groupes de travail, le fonctionnement des administrateurs généraux et le développement de l'organisation. 